\title{
Nordiques
}

\section{Les cours de langue maternelle et de culture d'origine pour enfants d'immigrés dans les systèmes scolaires des pays nordiques}

\section{Roger Marmus}

\section{CpenEdition}

Journals

Édition électronique

URL : http://journals.openedition.org/nordiques/509

DOI : $10.4000 /$ nordiques.509

ISSN : 2777-8479

Éditeur :

Association Norden, Bibliothèque de Caen la mer

\section{Édition imprimée}

Date de publication : 1 novembre 2018

Pagination : 59-76

ISBN : 9791095914020

ISSN : $1761-7677$

\section{Référence électronique}

Roger Marmus, "Les cours de langue maternelle et de culture d'origine pour enfants d'immigrés dans les systèmes scolaires des pays nordiques », Nordiques [En ligne], 36 | 2018, mis en ligne le 31 octobre 2020, consulté le 14 mars 2021. URL : http://journals.openedition.org/nordiques/509 ; DOI : https:// doi.org/10.4000/nordiques.509 


\section{Les cours de langue maternelle et de culture d'origine pour enfants d'immigrés dans les systèmes scolaires des pays nordiques}

Roger Marmus*

RÉSUMÉ

Pour permettre aux élèves issus de familles nouvellement arrivées de rester en contact avec leur langue et leur culture d'origine, et peut-être aussi pour faciliter leur intégration sociale, les pays nordiques ont mis en place, à différentes échelles, depuis environ cinq décennies, des cours en langue maternelle en parallèle avec les enseignements traditionnels. Dans les années 1960, ces classes étaient plus ou moins conçues comme une politique de promotion de la culture de l'Autre. Cinquante ans plus tard, alors que le contexte de l'accueil des migrants a changé et que le débat sur la question de l'identité est au centre des préoccupations, des voix se sont élevées pour remettre en question ces enseignements. Certains, en particulier au Danemark, critiquent leur prétendue inefficacité et les coûts financiers inutiles, d'autres les considèrent comme un emblème de la société multiculturelle à promouvoir. Après avoir étudié le rôle joué par ces enseignements dans le grand débat sur les langues et leur lien avec les politiques d'intégration culturelle, l'étude montre comment chacun des pays nordiques a géré les besoins et souhaits des écoles et des familles. L'enquête offre les éléments de réflexion pour comprendre quel pourrait être, dans les pays nordiques, le sort qui sera fait à ces cours, et par-delà, à l'idée de multiculturalisme institutionnalisé.

\section{ABSTRACT}

To enable pupils from newly arrived immigrant families to keep in touch with their native language and cultural background, and perhaps also to facilitate their social integration, the Nordic countries have implemented, at different scales, for about five decades, mothertongue courses that coexist with traditional teachings. This is a widely debated topic which illustrates different national policy strategies. These classes were initially, in the sixties, thought more or less as a policy of promoting the maintenance of diversity. Fifty years later, with the context of the reception of migrants that has changed, and the debate on identity at the forefront, voices are raised to question these teachings. Some, in particular in Denmark, criticize their supposed costly inefficiency, others claim them as an emblem of the multicultural society. After having studied the role played by these teachings in the great debate on languages and the link they have with the settlement policies, we will see how each of the Nordic countries has managed the needs and demands of education skills. We may then be able to predict the fate that will befall them, and to glimpse, in connection with this, the future of this kind of institutionalized multiculturalism in the Nordic countries.

* Roger Marmus est chercheur free-lance, diplômé d'un doctorat en études scandinaves de l'université de Paris-Sorbonne (Paris IV). Il réside à Göteborg, en Suède. 
Pour permettre aux écoliers issus de familles immigrées nouvellement arrivées de rester en contact avec leur langue et leur culture d'origine, et peut-être aussi de faciliter leur intégration, les pays nordiques ont mis en place, à différentes échelles, depuis environ cinq décennies, des cours de langue maternelle qui coexistent avec les enseignements traditionnels. Décriés par les uns pour leur supposée coûteuse inefficacité face aux objectifs de départ, vénérés par les autres comme un emblème de la société multiculturelle, ces cours soulèvent, au cœur du débat sur les réformes envisageables des systèmes éducatifs, des interrogations pédagogiques et politiques. Après avoir étudié le rôle que jouent ces enseignements dans le grand débat touchant aux langues et le lien qu'ils entretiennent avec les dispositions visant l'insertion des immigrés, nous verrons comment chacun des pays nordiques a su gérer les besoins et les demandes en la matière. Nous serons peut-être à même alors de pronostiquer, au-delà des questions de légitimité et d'opportunité, le sort qui leur sera fait, et d'entrevoir, en lien avec cela, l'avenir, dans les pays nordiques, du multiculturalisme institutionnalisé.

\section{1) LANGUe, ÉCOlE ET MUlTiCUlTURALISME : LES ENJEUX DU MULTILINGUISME INSTITUTIONNALISÉ}

Il ne se passe pas un jour sans que ne soit fait mention dans les différents médias nordiques de la nécessité d'agir en matière linguistique. Probablement parce que les pays du Septentrion représentent une part modeste de la population mondiale, et que les idiomes qui y sont parlés sont comme des livres rares à préserver. Il n'est pas exagéré de dire que ces pays ont développé à cet effet une sensibilité particulière aux langues, à leur préservation comme à leur diffusion. De la Finlande, qui vit au quotidien la cohabitation de deux parlers d'origines très éloignées, à l'Islande, qui maintient coûte que coûte une culture vernaculaire face à une américanisation rampante, tout en s'estimant, et se voulant, assez proche des pays frères pour privilégier l'apprentissage des autres langues scandinaves, en passant par la Norvège qui entretient la coexistence officielle de deux langues écrites, le bokmål et le nynorsk, on constate, dans l'ensemble de la région nordique, une diversité riche et choyée que ne vient pas démentir la présence des langues et dialectes des minorités autochtones (les langues sames), ou historiquement associées (le yiddish). Cette prospérité linguistique plus ou moins bien vécue, et finalement assez stable depuis un siècle, doit néanmoins être fortement nuancée et recontextualisée. En effet, depuis environ cinquante ans s'est imposée une autre configuration démographique qui a obligé les gouvernements à repenser la place des cultures allochtones dans le patrimoine commun à défendre. 
Avec des différences d'abord évidentes - la Norvège et le Danemark n’ont pas été des terres d'immigration dans l'immédiat après-guerre ! -, l'évolution migratoire suit, dans les pays scandinaves, une destinée semblable dans le dernier tiers $\mathrm{du} \mathrm{XX}^{\mathrm{e}}$ siècle. À des dates synchrones, les pouvoirs publics des pays respectifs décident de limiter les arrivées de main-d'œuvre. Les restrictions sont établies, au Danemark en 1969-1970, avant une fermeture complète du pays aux travailleurs en 1973 ; le même développement existe en Norvège, où des limites interviennent en 1971, précédant un arrêt total en $1975^{1}$. L'immigration a toutefois continué, surtout à partir des années 1980, sur la base du droit d'asile. On notera toutefois que, par la suite, le souhait a été de mettre un terme au courant migratoire venant des pays extracommunautaires (l'Europe étant ici entendue au sens large, en gros comme l'ensemble des états signataires de l'accord sur l'EEE - l'Espace économique européen -).

L'école, lieu par définition d'échanges et de transmissions culturels, s'est vue, comme d'autres institutions, mais probablement plus que d'autres, assigner la mission de maintenir la possibilité d'un dialogue entre les communautés s'exprimant dans des langues différentes et, ce faisant, d'organiser une société devenue de facto multiethnique. Au fur et à mesure des années, le modèle d'une orthodoxie assimilationniste à la française est rejeté. Les écoles nordiques, suivant des directives européennes des années 1970, mais également leurs propres choix politiques, ont donc mis en place, avec, nous allons le voir, des intensités et des amplitudes bien différentes, des enseignements qui offrent la possibilité d'un maintien, voire d'un progrès de la langue d'origine de l'élève. Voisinant avec les cours de mathématiques, de sciences naturelles, d'anglais, etc., apparaissent donc, à la fin des années 1960, et plus sérieusement dans la seconde moitié des années 1970, des cours de langue maternelle. Ainsi enseigne-t-on l'espagnol, l'arabe, le persan, etc., au gré des besoins apparus en complément des enseignements de la langue du pays hôte. Une politique scolaire bienveillante se met en place à l'endroit des filles et fils de travailleurs immigrés, alors que s'appliquent des politiques qui tendent à restreindre l'importation de main-d'œuvre : c'est en effet en 1966 que sont prises, en Suède, les premières mesures visant à limiter l'immigration. Cependant, il n'est pas faux de constater à propos de cette époque un double mouvement concomitant : le frein à l'immigration venant du sud, et le rejet d'une politique visant la trop contraignante assimilation ("À Rome fais comme les Romains !»). On choisit ainsi de donner aux nouveaux arrivants, et en l'occurrence à leurs enfants, le droit de conserver et de maîtriser leur culture. Pour ce qui est le cas de la Suède, le tournant reste l'année 1975, au cours de laquelle décision est prise, à l'égard

1 Grete Brochmann et Anniken Hagelund, "Migrants in the scandinavian welfare state. The emergence of a social policy problem ", Nordic Journal of Migration Research, n 1, 2011, p. 16. 
des immigrés et des minorités, d'une politique qui servira de base aux futures stratégies d'intégration. Trois grands principes sont mis en avant : jämlikhet («la parité »), samverkan (" la coopération») $)^{2}$ et puis, ce qui semble être le nœud central du dispositif voulu par Olof Palme, valfrihet («la liberté de choix») $)^{3}$. Les travailleurs immigrés sont censés vivre dans une égalité de droit avec leurs camarades suédois, doivent pouvoir compter sur leur assistance et intervenir dans les affaires de la cité, et puis surtout doivent avoir la liberté de décider dans quelle mesure ils souhaitent être intégrés dans la société d'arrivée. Même si le terme n'existe pas à l'époque, on peut lire dans le texte la forme aboutie d'une politique " multiculturaliste " qui avait été initiée à l'international par d'autres pays occidentaux, en particulier le Canada, l'Australie et la Nouvelle-Zélande ${ }^{4}$.

Les cours de langue d'origine, qui se révèlent dans ce contexte de restriction souhaitée des arrivées comme un signe du changement de paradigme quant à la question migratoire, vont être l'une des manifestations concrètes des principes de base des politiques d'accueil de 1975 et l'incarnation d'une liberté réelle de sauvegarder et d'entretenir ce qui constitue les parts essentielles d'une identité : la langue et la culture. Même si en pratique ces enseignements existaient déjà, la nouveauté est toutefois leur légitimité et le fait qu'ils fassent partie intégrante, en tout cas en Suède, des programmes scolaires.

L'idée d'une sauvegarde de la langue maternelle n'est pas nouvelle. Déjà en 1953, l'UNESCO prenait position dans un rapport sur l'emploi des langues vernaculaires dans l'enseignement ${ }^{5}$. Par " langue vernaculaire ", il fallait entendre la " langue maternelle d'un groupe socialement ou politiquement dominé par un autre groupe qui parle une langue différente $»^{6}$. Le rapport reprenait, entre autres, les conclusions d'une réunion d'experts qui s'était déroulée à Paris deux ans plus tôt, suite à la volonté de l'assemblée générale des Nations unies (résolution 329) de confier le soin à l'Organisation des Nations unies pour l'éducation, la science et la culture d' " entreprendre une étude d'ensemble " sur l'enseignement " des langues vernaculaires comme véhicule de l'enseignement dans les écoles $»^{7}$.

2 Le droit de vote dans les élections locales suédoises est institué la même année pour les immigrés installés depuis au moins trois ans.

3 Termes contenus dans le texte liminaire de la " proposition » 1975:26, votée par le Riksdag « uni » (enig).

4 Mats Wickström, The difference white ethnics made. The multiculturalist turn of Sweden in comparison to the cases of Canada and Denmark, in Migrations and welfare states. Policies, discourses and institutions, Helsinki, NordWel-Studies, 2013, p. 25-58, [https://www.researchgate.net/publication/303051284_ The_difference_white_ethnics_made_The_multiculturalist_turn_of_Sweden_in_comparison_to_the_ cases_of_Canada_and_Denmark].

5 UNESCO, L'emploi des langues vernaculaires dans l'enseignement. Monographies sur l'éducation de base, Paris, UNESCO, 1953, [http://unesdoc.unesco.org/images/0013/001315/131582fo.pdf].

6 Ibid., p. 52.

7 Ibid., annexe II, p. 156. 
Déjà à l'époque, les experts insistaient sur la logique qu'il y a, d'un point de vue pédagogique, à utiliser la langue maternelle. Le rapporteur, Aurélien Sauvageot, un normalien spécialiste des langues finno-ougriennes, et ses collègues auront toutefois le souci de faire état des objections possibles « qui ne leur semblent pas fondées $»^{8}$ :

a) Qu'en est-il lorsque les langues n'ont pas de grammaire ou d'alphabet ? b) Que faire lorsque l'enfant connaît déjà sa langue ${ }^{9}$ ?

Le rapport offre des réponses succinctes qui sont aisées à retenir :

a) "Nous posons en principe que rien, dans la structure d'une langue, quelle qu'elle soit, ne s'oppose à ce que cette langue devienne un véhicule de la civilisation moderne. $»^{10}$

b) «Il l'a suffisamment apprise pour ses besoins d'enfant, mais il lui faudra bien, à mesure qu'il avance en âge, élargir la connaissance qu'il en a. " ${ }^{11}$

Deux autres objections seront abordées dans le rapport ; elles méritent à notre sens qu'on s'y attarde, car elles résument les arguments qui seront, durant les décennies à venir, au cœur des critiques formulées par les adversaires de ces enseignements. Ces objections, pédagogique pour l'une, politique pour l'autre, couvrent le champ de la réalité selon les deux perspectives classiques : l'individuel (le développement de l'élève) et le collectif (la capacité à maintenir l'unité nationale).

La première critique ("L'emploi de la langue maternelle empêchera l'acquisition d'une seconde langue... $»^{12}$ ) doit être interprétée à l'aune de ce qui faisait spontanément dans les classes où régnait la peur de voir le nouvel élève subir une forme abâtardie du bilinguisme, c'est-à-dire le sort de ceux qui n'arriveront jamais à maîtriser parfaitement aucune langue, ni celle du pays d'origine, qui connaîtra une forme de dégénérescence, ni celle du pays d'accueil, qui sera toujours imparfaite - une situation que l'on nommera "semi-linguisme ${ }^{13}$. Il n'est pas interdit de penser que les experts (Aurélien Sauvageot en tête) avaient à l'esprit ce qui a pu se passer aussi pour les jeunes Finnois qui ont été évacués et recueillis dans des familles suédoises durant la Seconde Guerre mondiale et qui ont dû, à marche forcée, se mettre au suédois, langue germanique, comme on le sait, bien éloignée du tronc commun finno-ougrien.

8 Ibid., p. 55.

9 Ibid.

10 Ibid.

11 Ibid.

12 Ibid., p. 56.

13 Dans le contexte suédo-finnois, le concept de «semi-linguisme " (halvspråkighet) a été proposé par le chercheur suédois Nils Erik Hansegård dans un essai datant de 1968 : Tvåspråkighet eller halvspråkighet?, Stockholm, Aldus/Bonniers, 1968. 
La seconde ("L'emploi de la langue vernaculaire fait obstacle à l'unité nationale... $\left.{ }^{14}\right)$ nous intéresse au plus haut point, car elle est celle qui mettra les politiques au pied du mur. Faut-il en effet encourager chez un enfant, dans une famille, dans une communauté, la langue que l'on est censé perdre à terme ? La réponse rapidement évoquée se limite au bon sens et met en garde contre la nocivité probable des politiques "visant de manière absolue " l'usage de la nouvelle langue, au détriment de la langue vernaculaire (le texte parle également de "langue locale " ${ }^{15}$ ), et, à terme, de la cohésion sociale, certains risquant de "se retirer de la vie nationale" :

Il est indéniable que dans un pays unilingue la tâche des pouvoirs publics est plus aisée que dans un pays plurilingue. Il n'en résulte pas cependant qu'une législation ou une politique scolaire exigeant que la langue officielle soit tout le temps employée doit donner les mêmes résultats que l'unilinguisme proprement dit. [...] De toute façon, il semble bien que le meilleur instrument qui puisse servir l'intérêt national soit l'avancement optimum de l'éducation, et que celui-ci, à son tour, puisse être favorisé par l'emploi de la langue locale comme véhicule de l'enseignement, tout au moins au début du programme scolaire. $^{16}$

Les cours de langue maternelle ont eu la faveur des législateurs. En Scandinavie, comme ailleurs, ils s'appuient sur des textes qui incitent et organisent leur mise en œuvre. Au niveau continental, on ne peut manquer de mentionner la directive européenne du 25 juillet 1977 qui, dans son article 3, invitait les États membres à prendre en faveur des enfants des travailleurs migrants « [...] les mesures appropriées en vue de promouvoir, en coordination avec l'enseignement normal, un enseignement de la langue maternelle et de la culture du pays d'origine [...] ${ }^{17}$. Ce texte sert encore de base aux cours, même s'il faut bien sûr élargir et adapter la signification des termes employés. On notera avec un certain recul, voire un certain amusement, qu'il y a un peu plus de quarante ans, on parlait d'enfants de « travailleurs » migrants, et que les déplacements migratoires avaient surtout à voir avec les importations de main-d'œuvre.

En France, cette directive a été aussi suivie d'effets. Les ELCO (Enseignements de langue et de culture d'origine) existent dans les écoles, même s'ils sont

14 UNESCO, L'emploi des langues vernaculaires dans l'enseignement, op. cit., p. 56.

15 La présence des mots « langue locale » doit nous rappeler que l'époque de rédaction est encore celle de la colonisation, où le soutien aux langues d'origine était surtout vu dans le cadre d'une instruction donnée dans les pays excentrés et sous tutelle.

16 UNESCO, L'emploi des langues vernaculaires dans l'enseignement, op. cit., p. 56.

17 Directive 77/486/CEE du Conseil du 25 juillet 1977, visant à la scolarisation des enfants des travailleurs migrants, Journal officiel des Communautés européennes du 6 août 1977, p. 32, [https://eurlex. europa.eu/legalcontent/FR/TXT/PDF/?uri=CELEX:31977L0486\&from=FR]. 
peu ou mal connus. Le portail du ministère de l'Éducation nationale, Éduscol, fait le point sur ces enseignements dans une notice rapide. On y comprend que c'est surtout l'aspect linguistique qui est mis en valeur : la maîtrise de la langue maternelle étant suivant leurs termes "un préalable nécessaire à la réussite d'une langue seconde ${ }^{18}$. Souvent critiqués, ces cours, pourtant peu nombreux, ne touchant qu'une poignée de langues ${ }^{19}$, et d'élèves (92 500 enfants ${ }^{20}$, sont sur la sellette et dépendent d'un recrutement d'enseignants très aléatoire puisque issus d'une mise à disposition des gouvernements respectifs, ou d'un recrutement local par les consulats. Bruno Le Maire, ancien député UMP, a de longue date milité pour la suppression de ces enseignements, et l'on retiendra son intervention à la Chambre des députés, le 3 mars 2015, lors d'une question au gouvernement, à l'occasion de laquelle, s'appuyant sur les conclusions très sévères du Haut Conseil à l'intégration, il dénonçait leur maintien :

Le résultat est mauvais [...]. Cet enseignement n'a pas favorisé l'intégration, mais le repli sur soi. Il n'a pas favorisé le sentiment d'appartenance à la nation française, mais le communautarisme. Nous, nous estimons que pour les enfants issus de l'immigration, leur nation, c'est la France, leur langue, c'est la langue française, et leur culture, c'est la culture française. ${ }^{21}$

La diatribe de Bruno Le Maire prenait le contrepied de ce que recommande la Commission européenne dans plusieurs de ses brochures. On citera ainsi l'extrait d'une d'entre elles, en version anglaise, datant de mars 2015, qui, très explicitement, invoque les travaux de recherche pour rappeler les bienfaits des cours de langue maternelle pour les personnes concernées : "There is conclusive research evidence that learning mother tongues alongside the language of instruction enhances not only their mother tongue competences but also their competences in the language of instruction. $»^{22}$

18 [http://eduscol.education.fr/cid52131/enseignements-de-langue-et-de-culture-d-origine-elco. html].

19 Neuf pays sont concernés : l'Algérie, la Croatie, l'Espagne, l'Italie, le Maroc, la Serbie, la Tunisie et la Turquie.

20 Matthieu Deprieck, "Bruno Le Maire veut expulser de l'école les "langues et cultures d'origine” ", L'Express, publié le 3 mars 2015, mis à jour le 12 novembre 2015, [https://www.lexpress.fr/actualite/ politique/lr/bruno-le-maire-veut-expulser-de-l-ecole-les-langues-et-cultures-d-origine_1657272.html].

21 Notre transcription des propos. L'enregistrement de la question au gouvernement est consultable sur YouTube ; les mots y sont prononcés dans la première minute de l'intervention (entre 0 min 46 et 1 min 06), [https://www.youtube.com/watch?v=L-ZF2_xVcvE].

22 European Commission, Language teaching and learning in multilingual classrooms, Bruxelles, European Commission, 2015, p. 12. "Il existe des preuves décisives émanant de la recherche selon lesquelles l'apprentissage des langues maternelles, parallèlement à la langue d'enseignement, améliore non seulement les compétences en langue maternelle, mais également les compétences en langue d'enseignement. " Notre traduction. 


\section{2) LE STATUT DE LA LANGUE MATERNELLE DANS LES DIFFÉRENTES POLITIQUES SCOLAIRES NORDIQUES}

Ceux qui considèrent que l'espace nordique est loin d'être une unité homogène trouveront dans la question de l'enseignement des langues maternelles du grain à moudre, tant il est vrai que chaque pays a abordé la problématique à ses conditions et selon son agenda. Concernés à des degrés divers, les pays se distinguent quant aux mesures adoptées et développent des solutions qui se rapprochent sans jamais se confondre. Être concerné n'est pourtant pas le seul discriminant, la volonté et l'enthousiasme le sont tout autant.

\section{A) Les pays peu concernés par les cours de langue maternelle: l'Islande, la Finlande et la Norvège}

Sans y être hostiles, l'Islande, la Norvège et la Finlande sont en retrait sur la présence institutionnalisée des cours de langue maternelle. La raison en est simple : jusqu’à présent l'immigration a été relativement faible dans ces pays. Dans ces contextes, les langues étrangères ont été des cas à part et leur existence considérée comme une affaire si peu visible qu'elle n'a pas nécessité l'intervention de la puissance publique, leur traitement étant renvoyé dans la sphère privée. La langue d'origine devait rester une affaire familiale, soit, en pratique, d'initiative essentiellement parentale.

\section{a) L'Islande}

L'absence de communautés étrangères importantes en Islande a longtemps été une évidence et n'a pas fait débat. Outre la présence américaine durant la guerre froide, et mis à part le décompte de quelques " expatriés " arrivés sur place pour raisons professionnelles, ou sentimentales, on a longtemps peiné à reconnaître une présence immigrée de poids exigeant un traitement particulier de la culture de l'autre. C'est plutôt le contraire qui a prévalu, puisque depuis le Moyen Âge les Islandais étaient restés un peuple homogène. À chaque crise migratoire qu’a connue l'Europe durant ces trente dernières années, seule une poignée, souvent issue des Balkans, a fait le long voyage pour rejoindre le pays. La situation a fortement évolué durant ces dernières années et l'on remarque une nette progression de la population étrangère, en particulier polonaise.

Par exemple, on notera ainsi que, suite à la dernière crise de 2015, Reykjavik, répondant aux demandes du Haut-Commissariat des Nations unies, a consenti à ce qu'un nombre de personnes exilées de Damas et de ses environs s'installe dans le pays. Entre 2015 et 2017, l'Islande a accueilli 118 Syriens ${ }^{23}$. Même si

23 Voir article de La Dépêche, publié en ligne et mis à jour le 2 février 2017 (avec AFP), [https://www. ladepeche.fr/article/2017/02/02/2509538-1-islande-asile-arctique-pour-refugies-syriens.html]. 
l'on prend en compte le nombre très modeste de 350710 habitants de l'île en juin 2018, le chiffre des accueillis reste mineur en absolu, et à l'image de la présence étrangère encore contenue, 39570 résidents étrangers soit $11,28 \%$ de la population totale ${ }^{24}$, même s'il faut noter le remarquable accroissement de la population sur les cinq dernières années : l'Islande comptait 321857 habitants en $2013^{25}$ ! Ces constatations faites, on apprendra que la faible concentration d'immigrés n'explique pas à elle seule la timidité en matière de diversité linguistique à l'école. Une autre raison, bien mise en avant par les pouvoirs publics, reste le souhait, par esprit pragmatique, et par volonté politique, de relier le pays à ses racines historiques, à savoir la Scandinavie et, dans une certaine mesure, à l'Amérique. En parcourant la version anglaise du guide islandais des enseignements (curriculum), on remarque que l'accent est mis sur l'apprentissage de l'anglais, langue vue comme la lingua franca qui partage des éléments d'histoire avec l'islandais : "Iceland has old links with the Anglo-Saxon language, culture and history. Icelandic and English belong to the same branch of languages, and the history of the countries has been interwoven through the ages. ${ }^{26}$

L'État islandais, prenant acte que les contacts avec l'ancienne autorité sont toujours prégnants et intéressent à la fois la collectivité et les individus, fait du danois la seconde langue la plus apprise dans le cadre de l'école obligatoire. Viennent ensuite le suédois et le norvégien qui sont également enseignés, autant pour des raisons de proximité culturelle et historique évidentes, que par souci matériel de permettre aux élèves, et pour nombre d'entre eux futurs étudiants, de posséder les langues que l'on pratique dans les universités scandinaves. Les autres langues habituelles, comme l'allemand, l'espagnol, le français n'interviennent qu'à partir du lycée.

L'enseignement de la langue maternelle dans ce contexte reste passablement oublié et l'on note son absence en tant que discipline autonome dans les textes officiels qui régissent le système scolaire islandais. La stratégie suivie reste dépendante des contraintes matérielles attenantes à la faible population - comment réunir des classes d'élèves ? Comment recruter du personnel enseignant ? - et renvoie à l'initiative privée la mise en place de tels cours. On admettra toutefois que la problématique n'est pas totalement absente du dernier curriculum de 1991, qui voit la maîtrise de la langue maternelle comme un élément essentiel de

24 Chiffres selon Hagstofa (Bureau islandais des statistiques), [https://www.statice.is/publications/ news-archive/population/population-in-the-1st-quarter-2018/].

25 Source Hagstofa, [https://issuu.com/hagstofa/docs/icelandinfigures2014].

26 Ministry of Education, Science and Culture, The icelandic national curriculum guide for compulsory schools-with subjects areas, p. 125. "L'Islande a des liens anciens avec la langue, la culture et l'histoire anglo-saxonnes. L'islandais et l'anglais appartiennent à la même branche linguistique et à travers les âges, il y a enchevêtrement dans l'histoire de ces pays. " Notre traduction. 
l'estime de soi, du lien familial et de la transmission du patrimoine culturel. Il est bien prévu que les nouveaux arrivants pourront se voir proposer des soutiens dans leur langue d'origine (en mathématiques, en biologie, etc.), mais point de cours institués de langue d'origine. Toute la philosophie suivie apparaît dans le passage du texte qui indique que c'est avant tout aux familles de prendre en charge un tel apprentissage. Les écoles impliquées peuvent également tenter de trouver des aides extérieures auprès d'organismes rompus aux questions multiculturelles :

It is important for the self-image of pupils with a mother tongue other than Icelandic that they preserve their knowledge and skills in their own mother tongue. [...] A solid knowledge of the mother tongue strengthens family ties and bonds with cultural heritage. Therefore, it is essential that parents are made aware of the importance of the mother tongue for the pupil and that they are encouraged and supported in emphasising (sic) linguistic upbringing at home by nurturing the pupil's mother tongue. Parents and schools should, of course, seek assistance where they can find it, such as from institutions and individuals that are knowledgeable about the acquisition of a second language and multiculturalism. ${ }^{27}$

\section{b) La Finlande}

La situation de la Finlande ne se démarque pas du lointain voisin nordique que nous venons d'étudier. On y trouve certes une situation linguistique très éloignée, puisque c'est le bilinguisme officiel qui y règne (le finnois et le suédois), mais on est surpris de constater que les courbes et les figures sont similaires lorsqu'elles relatent l'histoire récente des migrations et incidemment la place des cours de langue maternelle dans les politiques scolaires.

Longtemps éloignée des questions migratoires, la Finlande fait l'expérience de l'immigration, depuis quelques années - essentiellement depuis la chute du communisme chez le grand voisin, la Russie, et depuis son entrée dans l'Union européenne. Entre 1990 et 2017, la part des immigrés dans la population est passée de $0,8 \%$ à $6,4 \%{ }^{28}$, avec des conséquences surprenantes sur les équilibres linguistiques. Ainsi, on observe que, depuis 2014, les langues officielles (finnois, suédois, same, le romani, la langue des signes) perdent du terrain quant au nombre de leurs pratiquants. En 2017, environ $7 \%$ de la population installée de manière permanente pratiquent d'autres langues (le russe, l'estonien et, dans une moindre

27 Ibid., p. 106. "Il est important pour l'estime de soi que les élèves de langue maternelle autre que l'islandais préservent leurs acquis et leurs compétences dans leur propre langue maternelle. [...] Une solide connaissance de la langue maternelle renforce les liens familiaux et les liens avec le patrimoine culturel. Il est donc essentiel que les parents prennent conscience de l'importance pour l'élève de la langue maternelle et soient encouragés à insister sur l'éducation linguistique (sic) à la maison et l'entretien de celle-ci. Les parents et les écoles devront bien entendu demander de l'aide là où ils peuvent la trouver, par exemple auprès d'institutions et de personnes connaissant bien les domaines de l'acquisition d'une langue seconde et du multiculturalisme. " Notre traduction.

28 Fred Karlsson, The languages of Finland, Turku, Lingsoft Inc., 2017, p. 78. 
mesure l'arabe, se taillent la plus grosse part du gâteau des langues). Ainsi, le suédois, parlé par 5,2\% de la population, perd sa situation privilégiée qu'il avait à côté du finnois (87,9\% de la population). Depuis 2014, les suédophones sont en infériorité numérique par rapport aux locuteurs d'autres langues (russes et autres). Ces derniers représentaient, en 2017, 6,8 \% des habitants ${ }^{29}$.

Face à cet afflux, la Finlande n'a pas changé du tout au tout sa vision du multilinguisme. Ayant pendant des années lutté pour maintenir un équilibre des langues entre les communautés, elle n'a pas à ce jour souhaité donner un statut officiel dans le milieu scolaire aux langues maternelles étrangères. Ici ou là, on compte bien sûr des cours, voire des classes qui intègrent les langues d'origine (environ 150 langues sont désormais parlées en Finlande), mais il ne s'agit pas de structures établies, dans un cadre programmatique ordonné ; on reste dans le domaine du facultatif. Les communes sont libres d'organiser des options l'aprèsmidi pour les enfants immigrés, avec l'aide éventuelle de l'État, mais la priorité reste donnée à la modersmål, entendue ici comme alternativement le suédois, le finnois, ou encore, mais rarement, le same ou le romani ${ }^{30}$.

\section{c) La Norvège}

Le rapport de force entre le bokmål et le nynorsk, à l'avantage du premier (environ $80 \%$ de la population), n'est peut-être plus la grande problématique de l'espace éducatif norvégien, car l'arrivée de migrants ces dernières décennies a changé pour une bonne part la donne. Il semble que, comme dans les exemples précédents, l'accès à des cours d'apprentissage et d'approfondissement dans sa propre langue soit marginalisé. Et lorsqu'ils existent, il apparaît que ces cours/ aides sont subordonnés à l'acquisition du norvégien (bokmål en pratique). C'est en tout cas ce qui se passe pour les garderies d'enfants qui, à l'occasion, peuvent intégrer dans le travail pédagogique une forme d'assistance linguistique. Le texte même du programme général d'enseignement semble vouloir ménager la chèvre et le chou, soit la culture de l'autre comme celle de l'autochtone. Chacun doit pouvoir s'y retrouver : "Les crèches doivent aider les enfants à utiliser leur langue

29 Fin 2017, la Finlande comptait 5513130 habitants, dont 373325 parlaient une langue étrangère. Le nombre de russophones s'élevait à 77 177, de pratiquants de l'estonien à 49590 et d'arabophones à 26 467. Source : Statistics Finland, [http://www.stat.fi/tup/julkaisut/tiedostot/julkaisuluettelo/yyti_ fif_201800_2018_19693_net.pdf].

30 Attention au faux ami ! Pendant longtemps, les Suédois ont utilisé le terme modersmål (mother tongue en anglais) pour signifier la langue autochtone parlée, c'est-à-dire tout simplement le suédois. Aujourd'hui, ceux-ci font allusion par ce même terme à la langue d'origine des enfants immigrés, ou issus des minorités locales. Les Finlandais suédophones ont gardé l'ancienne acception et l'utilisent dans leur documentation pour évoquer, selon les personnes, le finnois, le suédois, et non le russe ou l'arabe. 
maternelle tout en travaillant activement à la promotion des compétences en norvégien des enfants $»{ }^{31}$

S'agissant des élèves du primaire et du secondaire, la logique est la même aux yeux de la loi sur l'éducation ${ }^{32}$, qui met au second plan les cours de langue d'origine, et qui insiste sur le norvégien, ces premiers n'étant envisageables om nødvendig (que "si nécessaire »), non comme une nécessité immanente, mais comme une mesure-outil très pragmatique visant à assurer l'acquisition des savoirs et compétences scolaires en attente d'un niveau suffisant en norvégien :

Lélève a droit à une formation linguistique spéciale jusqu’à ce qu'il ait suffisamment de compétences en norvégien pour suivre l'enseignement ordinaire à l'école. Si nécessaire, l'élève a également droit à une formation professionnelle bilingue et à un enseignement en langue maternelle..$^{33}$

L'abandon de tels cours semble être en marche. Comment interpréter autrement l'absence des arguments phares qui présidaient au maintien de ce type d'études? Dans un ancien "plan de cours» (Kursplan) typique des années 1980, dit Lareplanen M87, était fait mention à la fois d'un lien de cause à effet bénéfique entre l'approfondissement de la langue d'origine et l'acquisition du norvégien, mais aussi d'un "bilinguisme fonctionnel " (funksjonell tospråklighet) : "Pour se sentir chez eux dans deux cultures, il faut que les élèves soient bilingues. L'école doit avoir pour objectif de développer leur bilinguisme fonctionnel. » ${ }^{34}$

Cette vision des choses a disparu dans le plan de cours depuis 1997, pour une perception plus intégrationniste, pour ne pas dire assimilationniste, dans l'actuel texte en vigueur datant de 2006 (Lareplanen Lk 06). Cette manière de procéder peut être comprise à la fois comme pédagogique et sociale, l'enfant étant ainsi supposé se préparer à court terme à se retrouver dans un bain monolingue

31 Document de travail concernant le " plan cadre " (Rammeplanen 2011), [https://www.udir. no/laring-og-trivsel/stottemateriell-til-rammeplanen/sprak-i-barnehagen--mye-mer-enn-bare-prat/3.spraktilegnelse/flerspraklige-barns-spraktilegnelse] : «Barnehagen må støtte barna når de bruker morsmålet sitt og samtidig arbeide aktivt med å fremme barnas norskspråklige kompetanse. " Notre traduction.

32 Loi sur l'enseignement primaire et secondaire, Lov om grunnskolen og den vidaregåande opplæringa (opplæringlova), [https://lovdata.no/dokument/NL/lov/1998-07-17-61/KAPITTEL_2\#\%C2\%A72-11].

33 Chapitre 2 sur l'enseignement primaire, $\$ 8$. Enseignement linguistique spécial pour les étudiants issus de minorités linguistiques, $\$ 2-8$, [https://lovdata.no/dokument/NL/lov/1998-0717-61/KAPITTEL_2\#\%C2\%A72-11] : "Sarskild språkopplaring for elevar frå språklege minoritetar ", "Eleven har rett til sarskilt språkopplaring inntil han eller hun har fätt tilstrekkelige norskferdigheter til a folge den ordinare opplaringen $i$ skolen. Om nødvendig har eleven også rett til tospräklig fagopplaring og morsmålsopplaring. " Notre traduction.

34 Ministère norvégien de l'Église et de l'Éducation (Norge Kirke-og undervisningsdepartement), Mønsterplan for grunnskolen M87, 1987, p. 38, [https://www.nb.no/nbsok/nb/2aef891325a059851965 d5b8ac193de5?lang=no\#39] : " En forutsetning for at elevene skal fole seg hjemme i to kulturer, er at de blir tospråklige. Skolen må ha som siktemål at elevene fär utvikle funksjonell tospråklighet. „ Notre traduction. 
norvégien, et à long terme prendre pleine part à une nation qui met un point d'honneur à défendre ses langues et dialectes nationaux ${ }^{35}$.

\section{B) La Suède : le "pays modèle"}

Avec la Suède, on s'approche du modèle idéal. Comme en d'autres domaines, le pays est allé loin dans ses choix, tant dans les textes que dans les moyens mis à disposition. Pour comprendre toute l'ampleur du projet, autant s'appuyer sur une constatation toute simple : la place des cours de langues d'origine dans le dernier plan de cours suédois datant de 2011 (aménagé à la marge pour mieux intégrer et favoriser les langues minoritaires). En ouvrant le document, disponible aussi sous forme de livre, on peut voir que les dispositions concernant l'enseignement des langues maternelles (modersmålsundervisning) sont mises au même plan que les mathématiques, les sciences naturelles, le suédois... La discipline est subordonnée à des intentions précises, des contenus et des exigences en matière de savoir.

Les années 1975 et 1977 sont les dates charnières dans l'histoire de leur apparition. C'est en effet à cette époque que les décisions sont prises, au niveau parlementaire, pour redynamiser ces cours qui existaient depuis 1968, et leur donner un fondement idéologique différent ${ }^{36}$. Comme nous l'avons vu plus haut, en introduction, la Suède d'Olof Palme souhaitait mettre un terme à la " politique de suédisation ${ }^{37}$ et réorienter la politique vers un multiculturalisme assumé. En matière éducative, on se dirigeait vers la promotion du bilinguisme. Comme pour marquer symboliquement le changement, la mesure sera accompagnée, en 1977, d'une nouvelle dénomination : de la modersmål (« langue maternelle »), on passera au terme hemspråk ( langue du foyer ») recouvrant la même réalité, mais censée mieux représenter l'aspect familial du concept. Faisant machine arrière, les pouvoirs publics, en 1997, imposeront de nouveau l'ancien terme modersmål, l'enjeu cette fois-ci étant d'en finir avec le malentendu qui avait pu naître et qui laissait penser que cette langue étrangère avait uniquement vocation à être parlée dans le contexte du foyer, et de mieux faire comprendre que, dans l'ordre des langues chez l'enfant, la langue maternelle arrive en première position ${ }^{38}$.

35 Maryann Jortveit, "Skolen i det flerkulturelle i Norge ", in På vandring og på fukt, Migrasjoni historisk perspektiv, B. E. Johnsen (éd.), Agder, Cappelen Damm Akademisk, 2016, p. 209-224, [https:// press.nordicopenaccess.no/index.php/noasp/catalog/book/22].

36 Pour un résumé en français du processus légal, nous renvoyons à la thèse de Piero Simeone Colla : L'héritage impensable. Conscience historique et technologies de l'identité dans la réforme éducative en Suède (1946-1980), thèse de doctorat de sociologie, soutenue le 2 mars 2017, École des hautes études en sciences sociales (EHESS), Paris, 2017, p. 719-726, [http://www.bononiadocta.it/PieroColla/Chap5. pdf].

37 Magnus Olsson et Armin Tröhler, Modersmålsundervisning och språkutveckling, mémoire de licence (C-Uppsast), Pedagogiska institutionen, Örebro Universitet, Örebro, 2006, p. 5.

38 Cité par ibid., p. 4. 
Même régulièrement remis en cause dans les médias par certains élus et certains intellectuels ${ }^{39}$, ces cours doivent se soumettre à des contraintes parfois difficiles à évaluer, comme celles qui obligent les potentiels élèves à justifier leurs acquis et une pratique régulière pour être admis (condition toutefois inexistante pour certaines langues minoritaires protégées, comme le meänkieli - finnois de Tornédalie -, le romi, le sami, le yiddish, ou la langue des signes), et à un volant d'heures d'enseignement laissé à l'appréciation des communes, qui comme on le sait sont, en Suède, en charge des questions scolaires. Une heure dans une commune, deux heures dans une autre, la durée des cours reste néanmoins modeste dans l'emploi du temps de l'élève.

Les années 1980 ont été le temps d'une remise à plat des ambitions et d'une prise de conscience des réalités sociales et économiques : par souci d'épargne, les enseignants n'ont plus vocation à être formés pour les besoins de la cause dans des universités ou des écoles normales (formations jugées trop coûteuses par les pouvoirs publics), les élèves ne sont plus autorisés à quitter les cours du matin pour leur rendez-vous hebdomadaire, ce qui oblige les enseignants, avec ce que cela implique de flexibilité de leur part, à organiser les cours uniquement l'aprèsmidi avec un minimum d'élèves légalement fixé à cinq.

Depuis la campagne électorale de 1990, et les succès du parti " populiste » $\mathrm{Ny}$ Demokrati, qui s'était ouvertement prononcé pour la suppression de cette matière, le débat est relancé et refait régulièrement surface dans les revues spécialisées ${ }^{40}$. Les partis conservateurs, enclins aux économies dans la dépense publique et le parti anti-immigration, Sverigedemokraterna, fondamentalement opposé à tout ce qui se rapproche du multiculturalisme, obligent les partisans du maintien à aiguiser les arguments et à diversifier les tâches pour justifier l'offre. De ce fait, les professeurs spécialisés n'ont plus uniquement leur propre langue comme objet d'étude, mais doublent souvent leur engagement d'une mission d'appoint dans les classes, ou en dehors, pour aider l'enfant à comprendre les autres matières. Ils deviennent concrètement à la fois interprètes, précepteurs, maîtres d'étude, soutien aux études (studiehandledare), missions pédagogiquement justifiées, mais qui s'éloignent des objectifs de départ.

39 C’est ainsi le cas d'Ebba Witt-Brattström, célèbre professeure de littérature, connue pour ses positions féministes tranchées et proches des milieux de gauche, qui, au nom d'une sauvegarde de la langue et du patrimoine culturel suédois, souhaitait dans un article du journal Dagens Nyheter (19 avril 2006) que l'on fasse préséance à la langue du pays.

40 Eva Annell, "Hur då modersmål ?", Magasin 360. Forskning och utveckling i skola och förskola, no 4, 2010, p. 18-20. 
Le gouvernement de gauche a commandé, au mois de janvier 2018, une étude d'évaluation au professeur Nihad Bunar, sociologue spécialiste de pédagogie de l'université de Stockholm, issu lui-même de l'immigration en tant que réfugié bosniaque. La plate-forme internet du gouvernement rapporte l'information en ouvrant son texte sur un argument développé par le ministre écologiste en fonction, Gustav Fridolin : "Les élèves qui ont la possibilité de développer leur langue maternelle apprennent en règle générale mieux le suédois et ont de meilleurs résultats à l'école. $»^{41}$

Les partisans peuvent jubiler en entendant les propos du ministre : les adversaires auront le contre-argument facile. Il s'agit d'une simple corrélation, et non la preuve d'un lien de cause à effet; de plus, il est admis que ce sont souvent les familles ambitieuses et assidues aux études, et ainsi plus enclines aux bons résultats, qui envoient leurs enfants dans ces cours. Le débat reste donc ouvert!

Une analyse plus attentive de l'évolution historique permet de constater que l'argument majeur des partisans s'éloigne de l'aspiration multiculturaliste de 1975, qui ambitionnait de combiner l'intégration dans la société suédoise avec le droit à sa culture d'origine, et laisse place à un argumentaire essentiellement pédagogique, et au fond assez consensuel, à l'heure où les résultats de l'enquête internationale d'évaluation des systèmes d'enseignement, PISA, qui place la Suède dans les profondeurs du classement, appellent à un sursaut.

\section{C) Le Danemark, foyer de dissidence}

Sur ce thème, le Danemark se démarque depuis longtemps de son voisin suédois. Il est difficile de nier que le sort réservé aux cours de langues maternelles est à l'image de la politique restrictive suivie en matière d'accueil et d'intégration des migrants, qui, par exemple, prévoit des tests de langue pour prétendre à un prolongement de séjour. L'arrivée au pouvoir, en 2002, d'un gouvernement de droite ayant décidé d'une politique plus radicale en matière d'intégration a mis un terme à la responsabilité de l'État dans l'organisation de ces cours, qui, jusqu'à cette date, étaient offerts en dehors des enseignements ordinaires, l'après-midi, voire le samedi. Aujourd'hui, ce sont les collectivités locales qui ont la liberté, mais aussi la charge financière, de décider la constitution de telles classes : un choix hautement sensible lorsque l'on sait que la presse à sensation, parfois sur ce point soutenue par des maires sociaux-démocrates, fait ses manchettes sur les dangers que feraient courir ces enseignements à l'intégrité culturelle du Danemark ${ }^{42}$. Sauvés

41 [https://www.regeringen.se/pressmeddelanden/2018/05/modersmalsundervisningen-skautredas/]. Notre traduction.

42 J. Normann Jørgensen, "Bilinguism and minority languages ", International Journal of the Sociology of Language, $\mathrm{n}^{\circ} 159$, février 2003, p. 75. 
par les obligations à l'égard des enfants de parents citoyens européens, ou issus de territoires dépendant de la couronne danoise (les îles Féroé, le Groenland) ${ }^{43}$, découlant de la loi appliquant la directive européenne 77/486 (évoquée ci-dessus), les cours financés publiquement se maintiennent, en particulier dans les quartiers dits sensibles des grandes villes, comme Copenhague ou Aarhus, lorsqu'un nombre suffisant d'enfants justifie la création d'une classe. Ces efforts demeurent relativement modérés lorsque l'on examine les chiffres de plus près, et que l'on constate que les élèves bilingues au Danemark ne représentent qu'une portion évaluée à 8-10\% des enfants scolarisés, ce qui se traduit en termes absolus par quelques milliers d'enfants concernés. Depuis le début des années 2010, certaines organisations non gouvernementales (l'Institut pour les droits de l'homme; le Comité des droits des enfants ; la Commission européenne contre le racisme et l'intolérance) se sont prononcées pour une révision des options prises, voire pour la réintroduction systématique de ces cours ${ }^{44}$.

La fronde vient en réalité d'en haut, du gouvernement même. Merete Riisager, membre du parti Liberal Alliance, et ministre de l'Éducation depuis 2016, n'y est ainsi pas allée de main morte en déclarant, en mai 2017 : «L'enseignement de la langue maternelle n'a pas d'effet [...]. Nous devons constater que c'est, sur le plan pédagogique, une impasse. ${ }^{45}$

La ministre s'appuyait sur les conclusions d'un rapport commandé par le ministère de l'Enseignement, paru en 2017, concernant une étude menée entre 2013 et 2015 dans une trentaine d'écoles, auprès de 6300 élèves de fin de primaire, sous la houlette du professeur Simon Calmar Andersen, de l'Institut des sciences politiques d'Aarhus ${ }^{46}$. Ce texte, tout en louant les effets "positifs» des cours de langue maternelle sur "le bien-être et la motivation de certains élèves ", s'en tenait (mis à part pour les élèves arabophones, chez qui un effet vertueux, visible dans les statistiques, existerait ${ }^{47}$ ) à une absence d'observation de l'efficacité des

43 La loi danoise 412 du 6 juin 2002 et le décret disposent que les enfants qui sont à la charge d'un résident du Danemark, ressortissant d'un autre État membre de l'Union européenne, ou d'un État couvert par l'accord sur l'Espace économique européen, peuvent prétendre à de tels cours. En outre, l'avis s'applique mutatis mutandis aux élèves parlant la langue féroïenne, ou groenlandaise.

44 Rapportdel'Institut for Menneskerettigheder, Danmarks Nationale Menneskerettighedsinstitution, Copenhague, 2015, p. 26, [https://menneskeret.dk/files/media/dokumenter/udgivelser/status/2014-15/ delrapporter/uddannelse.pdf].

45 Propos rapportés par plusieurs journaux : [https://jyllands-posten.dk/politik/ECE9548568/ minister-undervisning-i-modersmaal-er-en-blindgyde], [https://www.bt.dk/politik/minister-undervisningi-modersmaal-er-en-blindgyde] : " Modersmålsundervisning virker ikke [...]. Vi må konstatere, at modersmålsundervisning er en blindgyde rent padagogisk. " Notre traduction.

46 Modersmålsbaseret Undervisning -Modersmålsundervisning på 1.Klassetrin, Aarhus, Aarhus Universitet School of Business and Social Sciences, 2017, [https://www.emu.dk/sites/default/ files/170503-Samlet-rapport-modersmaalsundervisning-paa-1-klassetrin_1.pdf].

47 Voir les conclusions du rapport, ibid., p. 45. 
enseignements comme instrument propédeutique pour l'apprentissage du danois. Les experts appelaient néanmoins à des recherches plus poussées sur les élèves du secondaire, afin de se prononcer quant au réel impact sur le long terme.

Dans cette partie du Nord également, la question semble se focaliser sur l'aspect préparatoire des enseignements et sur leur capacité à consolider le danois, ainsi que toutes les matières enseignées dans cette langue. Ce paradoxe a été mis en évidence et dénoncé par une sociolinguiste danoise, Line Möller Daugaard, spécialiste des aspects ethnographiques du modersmålsundervisning (enseignement de langue maternelle) qui invoque un "droit propre " à exister accordé aux autres matières, mais apparemment récusé à ces cours : «Personne ne s'attend à ce que le cours d'allemand démontre sa valeur en s'appuyant sur un effet bénéfique prouvé sur les résultats aux tests de lecture en danois. $»^{48}$

\section{3) QUEL AVENIR POUR CES ENSEIGNEMENTS ?}

Face à l'américanisation des cultures et à l'anglicisation rampante des formes de communication visibles dans une bonne part de l'Europe, mais aussi face aux risques toujours inhérents de séparatisme, les pays nordiques se battent pour défendre leurs spécificités linguistiques. Ces particularismes ne touchent pas seulement les langues établies des peuples majoritaires, ils visent aussi ceux des ethnies ou des minorités autochtones qui peuvent justifier d'un ancrage historique bien ancien. Dans le cadre scolaire, on note l'existence dans chacun des pays nordiques concernés de mesures, lesquelles, visant à préserver la part d'altérité qui est en soi, organisent la survie des cultures autochtones, au prix parfois d'une sorte de " préférence nationale " qui prend la forme assumée d'une " discrimination positive ». On perçoit ainsi une sorte de concurrence entre les langues maternelles où certaines sont discrètement avantagées. L'exemple frappant reste le plan de cours des langues maternelles en Suède qui fait désormais la part belle aux langues minoritaires reconnues de Suède, non pour des raisons issues de la nature même de la langue (sa difficulté, la présence d'une alphabet différent, etc.), mais pour des raisons de légitimité historique, ou politique, au risque d'une distorsion sémantique sur la notion même de "langue maternelle ». Il découle des textes en vigueur que les familles où l'on parle le sami, le yiddish, le finnois ou le meänkieli peuvent inscrire leurs enfants sans que ceux-ci fassent état de prérequis, alors

48 Line Möller Daugaard et Martha Sif Karrebæk, Modersmålsundervisning. Flere sprog giver flere mulligheter, article du 22 juin 2016, sur le site en ligne d'informations scientifiques, Videnskab.dk., [https://videnskab.dk/en/node/21177] : "Selv skolens øvrige sprogfag har trods alt legitimitet i egen ret; ingen forventer, at tyskundervisning demonstrerer sin vardi gennem påviselig effekt på elevernes resultater $i$ danske lasetest. " Notre traduction. 
que l'ensemble des autres langues se voit interdire les cours à destination de purs débutants ${ }^{49}$. Est-il légitime de parler de la sorte de «langue maternelle » lorsqu'on ne la pratique pas?

Assiste-t-on à la fin d'un modèle de multiculturalisme institutionnalisé ? Il n'est pas sûr que les enseignements des langues d'origine aillent vers un avenir radieux. L'individualisme contemporain et l'autonomie des enfants qui servent la cause d'une identité choisie risquent de remettre en question les décisions en réalité souvent parentales; l'inflation des langues enseignées et du nombre d'élèves ${ }^{50}$ posent de nombreuses difficultés organisationnelles, comme financières, qui touchent en priorité les régions rurales. Les communes sont de plus en plus réticentes à entretenir des équipes pédagogiques perçues comme disproportionnées alors que les événements récents - comme la crise des migrants de 2015 - les ont sollicitées bien plus que d'habitude, et que les électeurs sont de plus en plus séduits par des partis qui prônent l'abandon pur et simple des cours à vocation multiculturelle. Alors quel sera le modèle alternatif pédagogico-culturel dans les écoles nordiques de demain qui conviendra aux opinions publiques? Pour certains, le modèle passera probablement par l'abandon d'une posture postcoloniale et par un approfondissement des ambitions de départ qui fasse la promotion des classes multilingues, voire " translangues " ${ }^{51}$ où les élèves locuteurs évolueront dans la classe en pratiquant une langue ici, en entendant une langue là, sans qu'un idiome soit en position d'infériorité ; pour d'autres, il se traduira par une reprise en main des règlements et des programmes, pour que ceux-ci, à terme, vantent les vertus universelles d'une découverte studieuse et d'une appropriation patiente des humanités, célébrées dans la langue du pays d'accueil.

49 Les mêmes dispositions favorables s'appliquent aux enfants adoptés, [https://www.skolverket.se/ regler-och-ansvar/ansvar-i-skolfragor/ratt-till-modersmalsundervisning].

50 Selon l'enquête du quotidien national suédois Svenska Dagbladet du 24 décembre 2017, le nombre d'élèves inscrits aux cours de langue maternelle s'élèverait, en Suède, à environ 160 000, soit près du double de celui qui existait dix ans auparavant, [https:/www.svd.se/dubbelt-sa-manga-barn-farmodersmalsundervisning].

51 Le concept de translanguaging, en vogue dans les universités anglo-saxonnes (et suédoises) préconise une forme d'alternance linguistique dans les classes multilingues : Ofelia García et Angel M. Y. Lin (dir.), Bilingual and Multilingual Education. Encyclopedia of Language and Education, Berlin, Springer, 2016, p. 2, [https://ofeliagarciadotorg.files.wordpress.com/2011/02/translanguaging-in-bilingual-education.pdf] : "The term translanguaging has been increasingly used in the scholarly literature to refer to both the complex and fluid language practices of bilinguals, as well as the pedagogical approaches that leverage those practices. " 